\title{
No evidence for rare recessive and compound heterozygous disruptive variants in schizophrenia
}

\author{
Douglas M Ruderfer ${ }^{1,2,3}$, Elaine T Lim ${ }^{4}$, Giulio Genovese ${ }^{3}$, Jennifer L Moran ${ }^{3}$, Christina M Hultman ${ }^{5}$, \\ Patrick F Sullivan ${ }^{6}$, Steven A McCarroll ${ }^{3,7}$, Peter Holmans ${ }^{8}$, Pamela Sklar ${ }^{1,9}$ and Shaun M Purcell ${ }^{\star, 1,2,3,4}$ \\ Recessive inheritance of gene disrupting alleles, either through homozygosity at a specific site or compound heterozygosity, \\ have been demonstrated to underlie many Mendelian diseases and some complex psychiatric disorders. On the basis of exome \\ sequencing data, an increased burden of complete knockout (homozygous or compound heterozygous) variants has been \\ identified in autism. In addition, using single-nucleotide polymorphism microarray data, an increased rate of homozygosity by \\ descent, or autozygosity, has been linked to the risk of schizophrenia (SCZ). Here, in a large Swedish case-control SCZ sample \\ (11 244 individuals, 5079 of whom have exome sequence data available), we survey the contribution of both autozygosity and \\ complete knockouts to disease risk. We do not find evidence for association with SCZ, either genome wide or at specific loci. \\ However, we note the possible impact of sample size and population genetic factors on the power to detect and quantify any \\ burden that may exist.
}

European Journal of Human Genetics (2015) 23, 555-557; doi:10.1038/ejhg.2014.228; published online 5 November 2014

\section{INTRODUCTION}

Schizophrenia (SCZ) is a highly heritable $\left(h^{2} \sim 0.6-0.8\right)$, debilitating disorder with an increasing number of implicated genetic loci ${ }^{1-7}$ from genome-wide association studies (GWAS) of common singlenucleotide polymorphisms (SNPs) and rare copy number variants (CNVs). Recently, large-scale exome-sequencing studies have searched for rare variants that strongly increase risk, ${ }^{8,9}$ although the relatively high baseline rate of rare neutral coding variation makes this challenging. ${ }^{10}$ To focus on rare variants that are a priori more likely to be highly penetrant for disease, one approach is to study de novo mutations, as these are effectively uncensored by selection pressures. ${ }^{11}$ A second strategy, adopted here, is to focus on the rare gene-disruptive variants, defined as nonsense, essential splice site or frameshift, where both copies of a gene are affected through homozygosity at a single site or compound heterozygosity (if an individual carries different disruptive variants on both the maternally and paternally derived copies of the gene). With respect to any one gene, we refer to these as 'complete knockout' variants. A recent study in autism reported complete knockout variants at a rate of 1 for every 20 healthy individuals ( 0.05 per person) with a twofold higher rate in cases. ${ }^{12}$

Whereas sequencing allows for direct discovery of rare complete knockout genotypes, rates of autozygosity (homozygosity by descent) can also be informative under recessive models. Previous studies have used SNP microarrays to identify multi-megabase runs of homozygosity $(\mathrm{ROH})$, that likely reflect autozygosity arising from a degree of recent inbreeding. ${ }^{13,14}$ A study of 9388, predominantly European, SCZ cases and 12456 controls demonstrated a significant positive correlation between the extent of $\mathrm{ROH}$ per individual and the disease risk. ${ }^{15}$ However, they did not identify any significant individual loci.

Here we apply both approaches in a large Swedish population-based sample ${ }^{8,16}$ of SCZ cases and controls: we first test the recessive model indirectly by comparing the SNP-derived $\mathrm{ROH}$ burden in cases versus controls, and second, we use exome sequencing to assess directly the burden of complete knockout variants.

\section{METHODS}

Sample ascertainment and data generation

Samples were collected from national registers in Sweden, cases were identified through the Swedish Hospital Discharge Register and controls were randomly selected from Swedish population registers. All subjects were appropriately consented and the Institutional Human Subject Committees approved the research. Sequencing was performed at the Broad Institute using Illumina (San Diego, CA, USA) GAII or HiSeq2000 machines. Exome capture was done using either version 1 or version 2 of the Agilent (Santa Clara, CA, USA) SureSelect Human All Exon Kit. Additional detail on sample ascertainment and sequence data generation has been previously published. ${ }^{8}$ BAM and VCF files are available in the dbGaP study phs000473.v1 (http://www.ncbi.nlm.nih.gov/ projects/gap/cgi-bin/study.cgi?study_id = phs000473.v1.p1).

\section{Runs of homozygosity calling}

We utilized previously published ${ }^{16}$ SNP imputation data of the entire Swedish data set representing a superset of the individuals that have been sequenced. We selected $\sim 55 \mathrm{k}$ SNPs in approximate linkage equilibrium from high-quality genotypes called from imputed dosages in 5001 cases and 6243 controls. We sought to detect $\mathrm{ROH}$ using PLINK ${ }^{17}$ with parameter settings $\mathrm{ROH}$ length $>1 \mathrm{Mb}$, at least one SNP per $100 \mathrm{~kb}$ and allowance of a single heterozygous event within a window of 50 SNPs, splitting calls if spanning $500 \mathrm{~kb}$ with no

\footnotetext{
${ }^{1}$ Division of Psychiatric Genomics, Department of Psychiatry, Icahn School of Medicine at Mount Sinai, New York, NY, USA; ${ }^{2}$ Institute for Genomics and Multiscale Biology, Icahn School of Medicine at Mount Sinai, New York, NY, USA; ${ }^{3}$ Stanley Center for Psychiatric Research, Broad Institute of MIT and Harvard, Cambridge, MA, USA; ${ }^{4}$ Analytic and Translational Genetics Unit, Psychiatric and Neurodevelopmental Genetics Unit, Massachusetts General Hospital, Boston, MA, USA; ${ }^{5}$ Department of Medical Epidemiology and Biostatistics, Karolinska Institutet, Stockholm, Sweden; ${ }^{6}$ Department of Genetics, University of North Carolina, Chapel Hill, NC, USA; ${ }^{7}$ Department of Genetics, Harvard Medical School, Boston, MA, USA; ${ }^{8}$ Medical Research Council Centre for Neuropsychiatric Genetics and Genomics, Institute of Psychological Medicine and Clinical Neurosciences, Cardiff University, Cardiff, UK; ${ }^{9}$ Friedman Brain Institute, Icahn School of Medicine at Mount Sinai, New York, NY, USA

${ }^{*}$ Correspondence: Dr SM Purcell, Department of Psychiatry, Icahn School of Medicine at Mount Sinai, 1 Gustave L. Levy PI, New York, NY 10029, USA. E-mail: shaun. purcell@mssm.edu
}

Received 10 March 2014; revised 16 July 2014; accepted 19 September 2014; published online 5 November 2014 
marker. This method and parameter selection represents those suggested in the literature after a careful comparison ${ }^{18}$ and is very similar to the approach used previously on a subset of these data. ${ }^{15}$

\section{Compound heterozygous identification}

To evaluate the role of complete knockout variants in SCZ we implemented a method to identify these variants within the framework of the statistical package Plink/Seq (http://research.mssm.edu/statgen/software.html). The method analyzes each transcript independently, ensuring that both the disruptive variants are affecting the same protein. As most pairs of variants will not be within the distance necessary to phase (length of sequencing read), we employed a previously used statistical phasing approach to identify compound heterozygous events. ${ }^{12}$ We defined compound heterozygosity as an individual carrying a heterozygous genotype on each chromosome at different locations within the same gene.

\section{Assessing significance using permutation}

Significance of complete knockout variants was assessed by permuting individual alleles independently assuming Hardy-Weinberg equilibrium (HWE). Permutation was performed within 296 groups of individuals matched on IBS from GWAS data to control for subtle population substructure (average number of individuals per group $=17$ ). We assessed the significance of gene sets by summing across multiple variants assuming independence.

\section{RESULTS}

Among 5001 cases and 6243 controls, we identified $4117 \mathrm{ROH}$ in 2001 individuals; $16 \%$ of individuals carried at least one $\mathrm{ROH}$. The average length of $\mathrm{ROH}$ was $8 \mathrm{Mb}$ (median length of $6.8 \mathrm{Mb}$ ) and ranged from 1.6 to $55.2 \mathrm{Mb}$. We also quantified each individual's genome-wide degree of homozygosity using Wright's inbreeding coefficient $(F)$. After including the first 10 multidimensional scaling (MDS) components to account for ancestry, homozygosity was similar in cases and controls as assessed by the number of $\mathrm{ROH}$ ( 0.38 cases, 0.32 controls, $P=0.60)$, total kilobases of $\mathrm{ROH}(3123 \mathrm{~kb}$ cases, $2544 \mathrm{~kb}$ controls, $P=0.88)$ or $F(0.0026$ cases, 0.0029 controls, $P=0.09$, Table 1$)$.

We next looked for complete knockout variants in a subset of 2477 cases and 2481 controls for whom exome sequence data were available. ${ }^{8}$ We identified 462 complete knockout rare (minor allele frequency $<5 \%$ ) genotypes at 193 genomic locations, a rate of 0.09 per person with no increased burden in cases (229 case; 233 control, Table 2). Of these, 405 were homozygous and 57 were compound heterozygous, with no significant enrichment in either class. Analysis of nonsynonymous and silent complete knockout variants yielded similar null results (data not shown).

Testing each site individually for association with increased risk (one-sided), 30 (16\%) had $P<0.05$ but none surpassed the Bonferroni correction for multiple testing (Supplementary Table S1). This excess of nominally significant tests is a product of testing only those sites carrying at least one complete knockout genotype: simulating null genotype data under HWE using the observed allele frequencies, we

Table 1 Summary of ROH and inbreeding coefficient $(F)$ in full Swedish GWAS sample, $P$-values are from a logistic regression with 10 MDS components and wave as covariates

\begin{tabular}{lccc}
\hline & Cases & Controls & P-value \\
\hline$N$ & 5001 & 6243 & \\
ROH rate & 0.38 & 0.32 & 0.60 \\
ROH $(k b)$ & 3123 & 2544 & 0.88 \\
$F$ & 0.0026 & 0.0029 & 0.09 \\
\hline
\end{tabular}

found a similar proportion of nominally significant sites when following a similar analytic procedure (data not shown).

For the X chromosome, males and females were analyzed separately. A single hemizygous disruptive variant in males results in a complete loss of gene function as in the autosomal complete knockout scenario. Using a minor allele frequency cutoff of $0.25 \%$, we observed 49 disruptive sites outside of the pseudoautosomal regions, a burden of 41 in cases and 41 in controls (one sided Fisher's exact test $P=0.5$ ). No individual site achieved nominal significance (Supplementary Table S2).

Finally, we selected gene sets previously demonstrated to have a significant polygenic burden of rare disruptive alleles (primarily observed as a single heterozygous genotype) in the same set of individuals. $^{8}$ Across 2546 genes in seven sets, we identified 35 complete knockout or male X chromosome disruptive genotypes, 19 in cases and 16 in controls $(P=0.38$; Supplementary Table S3). Although the set of genes carrying disruptive de novo alleles in SCZ displayed nominal significance $(4: 0$, one-sided $P=0.027)$, this does not survive correction for the seven tests. We also considered genes with known syndromic autosomal recessive causes of autism or intellectual disability. ${ }^{19}$ Three case genotypes and five control genotypes were found across 119 intellectual disability genes and one case genotype and zero control genotypes were found across 28 autism genes; neither test reached nominal significance $(P$ intellectual disability $=0.72, P$ autism $=0.57$.

\section{DISCUSSION}

Overall, we did not observe a significant enrichment of ROH in SCZ cases. In a larger sample (21 844), Keller et al. ${ }^{15}$ reported a significantly higher burden of $\mathrm{ROH}$ in SCZ cases, suggesting that the current study may be under-powered. We note that the Keller et al. study included a small proportion of the Swedish individuals reported here, although the authors did not find evidence for increased $\mathrm{ROH}$ in the Swedish subset, consistent with our results. This suggests that population genetic and ancestry characteristics of a sample could be a further determinant of the relative role of rare recessive variants in the risk for SCZ.

The amount of recent inbreeding will contribute to the levels of homozygosity present in a population. Our sample was ascertained from Sweden, but includes a proportion of individuals of Finnish descent. Utilizing populations with recent inbreeding will increase the frequency of these events and the power to detect enrichment, an approach that has been used successfully for CNVs. ${ }^{20}$

In conclusion, this sample does not lend support for a major role of disruptive recessive or compound heterozygous variants in the risk of SCZ. There was no overall burden of $\mathrm{ROH}$ genome wide or of complete knockout variants exome wide, no specific genomic site was significantly associated and no gene achieved significance after correction. These results largely mirror those of de novo SCZ trio studies (Fromer et al. ${ }^{11}$ ), in which there is no significantly increased

\section{Table 2 Summary of two-hit disruptive genotypes}

\begin{tabular}{lcc}
\hline & Case & Control \\
\hline$N$ & 2477 & 2481 \\
Homozygous & 200 & 205 \\
Compound heterozygous & 29 & 28 \\
All two-hit & 229 & 233 \\
Rate & 0.09 & 0.09 \\
\hline
\end{tabular}


burden overall and very few individual genes are unambiguously implicated, although for de novo mutations, broader effects can be seen across subsets of functionally-related genes. Rare recessive and compound heterozygous sites may still have a role in disease risk although larger samples or different analytic approaches may be required.

\section{CONFLICT OF INTEREST}

The authors declare no conflict of interest.

1 Bassett AS, Chow EW, AbdelMalik P, Gheorghiu M, Husted J, Weksberg R: The schizophrenia phenotype in 22q11 deletion syndrome. Am J Psychiatry 2003; 160: 1580-1586.

2 International Schizophrenia Consortium: Rare chromosomal deletions and duplications increase risk of schizophrenia. Nature 2008; 455: 237-241.

3 Ripke S, Sanders AR, Kendler KS et al: Genome-wide association study identifies five new schizophrenia loci. Nature genetics 2011; 43: 969-976.

4 O'Donovan MC, Craddock N, Norton N et al: Identification of loci associated with schizophrenia by genome-wide association and follow-up. Nat Genet 2008; 40: 1053-1055.

5 Walsh T, McClellan JM, McCarthy SE et al: Rare structural variants disrupt multiple genes in neurodevelopmental pathways in schizophrenia. Science 2008; 320: 539-543.

6 McCarthy SE, Makarov V, Kirov G et al: Microduplications of $16 \mathrm{p} 11.2$ are associated with schizophrenia. Nat Genet 2009; 41: 1223-1227.
7 Lichtenstein P, Yip BH, Bjork C et al: Common genetic determinants of schizophrenia and bipolar disorder in Swedish families: a population-based study. Lancet 2009; 373: 234-239.

8 Purcell SM, Moran JL, Fromer M et al: A polygenic burden of rare disruptive mutations in schizophrenia. Nature 2014; 506: 185-190.

9 Kiezun A, Garimella K, Do R et al: Exome sequencing and the genetic basis of complex traits. Nat Genet 2012; 44: 623-630.

10 Zuk O, Schaffner SF, Samocha K et al: Searching for missing heritability: Designing rare variant association studies. Proc Natl Acad Sci USA 2014; 111: E455-E464.

11 Fromer M, Pocklington AJ, Kavanagh DH et al: De novo mutations in schizophrenia implicate synaptic networks. Nature 2014; 506: 179-184.

12 Lim ET, Raychaudhuri S, Sanders SJ et al: Rare complete knockouts in humans: population distribution and significant role in autism spectrum disorders. Neuron 2013; 77: 235-242.

13 Lencz T, Lambert C, DeRosse P et al: Runs of homozygosity reveal highly penetrant recessive loci in schizophrenia. Proc Natl Acad Sci USA 2007; 104: 19942-19947.

14 Power RA, Keller MC, Ripke $S$ et al: A recessive genetic model and runs of homozygosity in major depressive disorder. Am J Med Genet B Neuropsychiatr Genet 2014; 165: 157-166.

15 Keller MC, Simonson MA, Ripke S et al: Runs of homozygosity implicate autozygosity as a schizophrenia risk factor. PLoS Genet 2012; 8: e1002656.

16 Ripke S, O'Dushlaine $\mathrm{C}$, Chambert $\mathrm{K}$ et al: Genome-wide association analysis identifies 13 new risk loci for schizophrenia. Nat Genet 2013; 45: 1150-1159.

17 Purcell S, Neale B, Todd-Brown K et al: PLINK: a tool set for whole-genome association and population-based linkage analyses. Am J Hum Genet 2007; 81: 559-575.

18 Howrigan DP, Simonson MA, Keller MC: Detecting autozygosity through runs of homozygosity: a comparison of three autozygosity detection algorithms. BMC Genomics 2011; 12: 460.

19 Betancur C: Etiological heterogeneity in autism spectrum disorders: more than 100 genetic and genomic disorders and still counting. Brain Res 2011; 1380: 42-77

20 Morrow EM, Yoo SY, Flavell SW et al: Identifying autism loci and genes by tracing recent shared ancestry. Science 2008; 321: 218-223.

Supplementary Information accompanies this paper on European Journal of Human Genetics website (http://www.nature.com/ejhg) 